\title{
Antimicrobial susceptibility testing of mycoplasmas by ATP bioluminescence
}

\author{
D. I. LIMB, P. F. WHEAT, J. G. M. HASTINGS* and R. C. SPENCER
}

Departments of Bacteriology, Royal Hallamshire Hospital, Glossop Road, Sheffield S10 2JF and *Clinical Microbiology, Queen Elizabeth Hospital, Edgbaston, Birmingham B15 2TH

\begin{abstract}
Summary. The susceptibility of 72 mycoplasmas to a range of antimicrobial agents was assessed in a 6-h ATP bioluminescence system. ATP was assayed with the Amerlite Analyser. Correlation with conventionally determined MICs was excellent for erythromycin and tetracycline even at $3 \mathrm{~h}$. However, for ciprofloxacin, correlation was poor unless incubation was extended to $6 \mathrm{~h}$.
\end{abstract}

\section{Introduction}

Mycoplasmas have been implicated as the cause of genital and respiratory tract infections and of lifethreatening infections in neonates. ${ }^{1}$ Standard antibiotic therapy for mycoplasmal infections includes tetracycline and erythromycin. ${ }^{2}$ However, Mycoplasma hominis is resistant to erythromycin and tetracycline is contra-indicated in certain clinical conditions. Furthermore, there has been an increasing number of reports of tetracycline-resistant strains of both $M$. hominis and Ureaplasma urealyticum. ${ }^{3,4}$ These problems have indicated the need for a rapid, practical means for determining antimycoplasmal susceptibility. The use of the luciferin-luciferase bioluminescence adenosine triphosphate (ATP) assay for the estimation of microbial biomass is well established. ${ }^{5} \mathrm{We}$ have described previously a protocol for the rapid antimicrobial susceptibility testing of Enterobacteriaceae ${ }^{6}$ and gram-positive cocci ${ }^{7}$ based on ATP bioluminescence. This protocol has now been modified for testing the susceptibility of mycoplasmas.

\section{Materials and methods}

\section{Organisms}

A total of 72 clinical isolates of $M$. hominis (36 strains) and $U$. urealyticum (36 strains) was tested. They were identified by arginine hydrolysis and production of urease respectively. ${ }^{1}$

\section{Antimicrobial agents}

Ciprofloxacin (Bayer), ofloxacin (Hoechst), fleroxacin (Roche), tetracycline (Lederle) and erythromycin (Abbott) were supplied as standard powders and solutions were freshly prepared before each experiment.

Received 20 Nov. 1989; revised version accepted 20 Nov. 1990.

\section{Determination of minimum inhibitory concentrations (MICs)}

Antimycoplasmal activity in the range $0.06-32 \mathrm{mg} /$ $\mathrm{L}$ was determined by the agar-dilution method $^{8,9}$ with Mycoplasma Agar (Oxoid) containing yeast extract $20 \% \mathrm{w} / \mathrm{v}$, and supplemented with horse serum $10 \%$ $\mathrm{v} / \mathrm{v}$ and $3 \mathrm{~mm}$ urea (filter-sterilised $1 \mathrm{~m}$ urea $0.3 \mathrm{ml} /$ $100 \mathrm{ml}$ of agar medium) for ureaplasmas. Inocula of c. $10^{7} \mathrm{cfu} / \mathrm{ml}\left(c .10^{4} \mathrm{cfu} / \mathrm{spot}\right)$ were transferred to the antibiotic-containing agar plates by means of a multipoint inoculator (Denley Instruments) and incubated for $72 \mathrm{~h}$ at $37^{\circ} \mathrm{C}$ in an anaerobic environment $\left(\mathrm{CO}_{2} 10 \%, \mathrm{H}_{2} 10 \%, \mathrm{~N}_{2} 80 \%\right)$. Ureaplasma colonies were visualised by spraying the plates with calcium chloride-urea stain. ${ }^{10}$ The plates were then examined by light microscopy with a low power objective ( $x$ 10). The MIC was defined as the lowest concentration of antimicrobial agent that inhibited visible growth.

\section{Microbial growth curve studies}

A 24-h broth culture of $M$. hominis was resuspended in equal volumes of Mycoplasma Broth (Oxoid) supplemented with horse serum $10 \% \mathrm{v} / \mathrm{v}$ and yeast extract $20 \%$ w/v with or without antimicrobial agent. The concentrations of antimicrobial agents were ciprofloxacin 4 and $8 \mathrm{mg} / \mathrm{L}$, erythromycin $4 \mathrm{mg} / \mathrm{L}$ and tetracycline $4 \mathrm{mg} / \mathrm{L}$. ATP measurements were assessed at the start of incubation and after incubation for 3, 6 and $9 \mathrm{~h}$ at $37^{\circ} \mathrm{C}$.

\section{Susceptibility testing by ATP bioluminescence}

Preliminary studies showed that 18-24-h broth cultures of $M$. hominis and $U$. urealyticum were suitable as starting inocula $\left(c .10^{6}-10^{7} \mathrm{cfu} / \mathrm{ml}\right)$. ATP measurements were assessed in the control and in broths containing $4 \mathrm{mg} / \mathrm{L}$ of ciprofloxacin, erythromycin or tetracycline at the start of incubation and after incubation for 3 and $6 \mathrm{~h}$ at $37^{\circ} \mathrm{C}$. 
Assay of ATP. A $200-\mu$ l sample of the culture was mixed with an equal volume of extraction reagent consisting of trichloroacetic acid $2.5 \%$ and $4 \mathrm{mM}$ ethylene diamine tetra-acetic acid. After $2 \mathrm{~min}, 20 \mu \mathrm{l}$ of this extract was added to $100 \mu \mathrm{l}$ of buffer (TrisEDTA) and $50 \mu \mathrm{l}$ of ATP Monitoring Reagent (luciferin-luciferase; LKB Wallac) in one well of a microtitration tray. Light emission was measured with an Amerlite analyser (Amersham International, Buckinghamshire). ATP bioluminescence was expressed as a percentage:

$\frac{\text { luminescence in broth with antimicrobial agent }}{\text { luminescence in control broth }} \times 100 \%$

From our previous experience of this assay, we categorised the results empirically as sensitive ( $\leqslant$ $40 \%$ ), intermediate $(41-49 \%)$ or resistant $(\geqslant 50 \%)$.

\section{Analysis of ATP and MIC susceptibility results}

ATP assays were compared with MIC values by error boxes (fig. 1), based on a method we have previously described. ${ }^{7}$ For strains in boxes A and B, the tests were considered to show agreement, for strains in box $\mathrm{C}$ a major disagreement and for strains in box $\mathrm{D}$ a very major disagreement. Strains for which the MIC did not differ by more than one dilution from the chosen concentration of antimicrobial agent in the ATP method were classified as showing agreement.

\section{Results}

MIC values determined conventionally are summarised in the table. All strains of $U$. urealyticum were

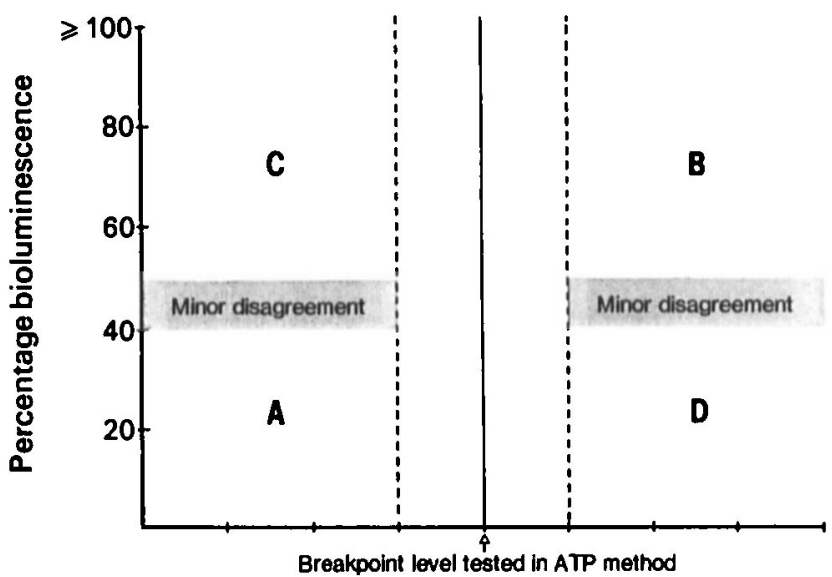

Minimum inhibitory concentration $(\mathrm{mg} / \mathrm{l})$

\section{(two-fold dilutions)}

Fig. 1. Comparison of ATP bioluminescence results and MIC values by error box analyses; box $\mathrm{C}$ represents major disagreement and box $\mathrm{D}$ very major disagreement.
Table. MIC values of quinolones, erythromycin and tetracycline for mycoplasmas

\begin{tabular}{llrrc}
\hline \multirow{3}{*}{ Species (n) } & Antimicrobial & \multicolumn{3}{c}{ MIC (mg/L) } \\
\cline { 3 - 5 } & & $50 \%$ & $90 \%$ & Range \\
\cline { 3 - 5 } M. hominis (36) & Ciprofloxacin & 1 & 1 & $0 \cdot 5-2$ \\
& Ofloxacin & 2 & 2 & $1-2$ \\
& Fleroxacin & 2 & 2 & $1-2$ \\
& Erythromycin & $>32$ & $>32$ & $>32$ \\
& Tetracycline & 0.5 & 32 & $0 \cdot 25-32$ \\
U. urealyticum (36) & Ciprofloxacin & 16 & 32 & $8-32$ \\
& Ofloxacin & 2 & 4 & $1-8$ \\
& Fleroxacin & 4 & 4 & $2-4$ \\
& Erythromycin & 2 & 2 & $2-4$ \\
& Tetracycline & 1 & 2 & $1-2$ \\
\hline
\end{tabular}

susceptible to erythromycin and tetracycline. In contrast, all strains of $M$. hominis were resistant to erythromycin. The majority of $M$. hominis strains were susceptible to tetracycline; five strains were resistant. Of the three fluoroquinolone agents, ciprofloxacin proved to be the most active against $M$. hominis but the least active against $U$. urealyticum.

\section{Mycoplasmal growth curve studies}

Growth of $M$. hominis could be detected by ATP bioluminescence after incubation for only $3 \mathrm{~h}$ in the antibiotic-free broth (fig. 2). At this time resistance to erythromycin and sensitivity to tetracycline could be demonstrated. In contrast, when ATP levels were measured in the broth containing ciprofloxacin 4 $\mathrm{mg} / \mathrm{L}$ after incubation for $3 \mathrm{~h}$, levels parallelled those of the control broth and only decreased after incubation for a further $3 \mathrm{~h}$ (fig. 2). The broth containing ciprofloxacin $8 \mathrm{mg} / \mathrm{L}$ showed no initial increase in ATP readings compared to the control.

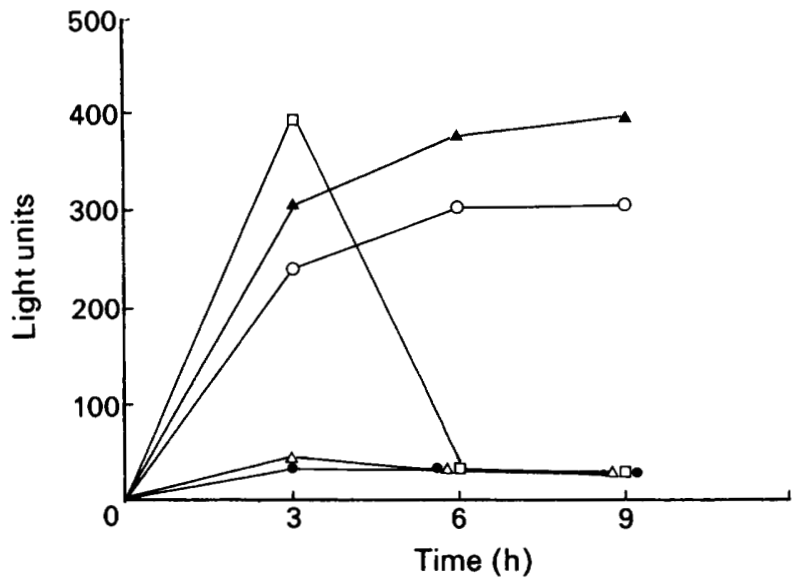

Fig. 2. Growth of $M$. hominis assessed by ATP bioluminescence: (A) control; (O) with erythromycin $4 \mathrm{mg} / \mathrm{L}$; (O) with tetracycline $4 \mathrm{mg} / \mathrm{L}$; $(\square)$ with ciprofloxacin $4 \mathrm{mg} / \mathrm{L}(\triangle)$ with ciprofloxacin $8 \mathrm{mg} / \mathrm{L}$. 


\section{Comparison of MIC values and ATP results}

Overall correlation between MIC values and ATP bioluminescence results was excellent (fig. $3 a-c$ ). There were no instances of major or very major disagreement. Predictably, mycoplasma strains tested against ciprofloxacin $4 \mathrm{mg} / \mathrm{L}$ required incubation for $6 \mathrm{~h}$ before satisfactory results were obtained (fig. 3c).

\section{Discussion}

Present antimicrobial susceptibility testing of mycoplasmas is cumbersome and time-consuming, and the end-point determination may be very subjective. The small size of these organisms $(c .0 \cdot 3 \mu \mathrm{m})$ and low maximum harvests of cells $\left(c .10^{7}-10^{8}\right.$ organisms $\left./ \mathrm{ml}\right)$ make microscopic and turbidimetric measurements unreliable. ${ }^{11}$

ATP is ubiquitous in all living cells and its assessment is an established method for measuring microbial biomass. ${ }^{5}$ This is particularly useful for enumeration of fastidious micro-organisms. In the present study, a rapid susceptibility method for mycoplasmas and ureaplasmas showed excellent correlation with conventional techniques, which are seldom performed on a routine basis in the laboratory. However, before correlation was acceptable for ciprofloxacin, incubation had to be extended to $6 \mathrm{~h}$. This may be similar to a phenomenon seen when Escherichia coli is exposed to ciprofloxacin - the organism shows initial filamentation followed by eventual lysis. ${ }^{12}$ Studies in our laboratory have shown that E. coli exposed to ciprofloxacin for only a few minutes yielded markedly increased levels of ATP. ${ }^{13}$

Stemler et al.$^{11}$ showed that ATP estimation could be used as a means of measuring the growth of U. urealyticum. They described a single-chamber luminometer which utilised larger volumes of reagents than were used in our study. We used a novel luminometer which worked to a microtitration forma ${ }^{13}$-this allows a reduction in reagent volume and facilitates the use of multi-channel dispensers for transfers and additions. ${ }^{7}$ The protocol described in this study is inexpensive and suitable for use in the diagnostic microbiology laboratory. However, further improvements are necessary if the technique is to be used on a routine basis. We are developing an extraction method in which all steps are performed to a microtitration format, and the addition of a microcomputer database would assist the storage and retrieval of susceptibility data.

This study was supported by a grant from Bayer UK Ltd, Newbury, Berkshire.
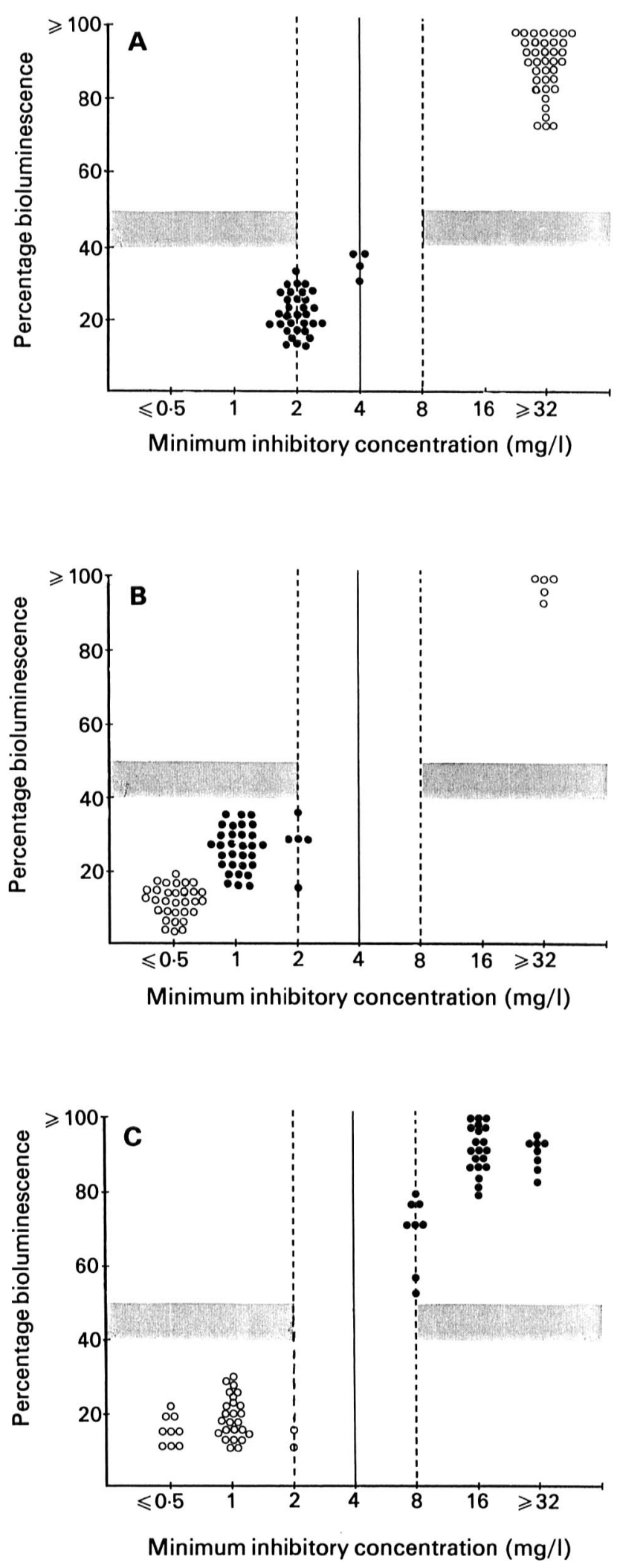

Fig. 3. Comparison of MIC results (mg/L) with 6-h ATP bioluminescence values for 36 strains each of $M$. hominis $(O)$ and U. urealyticum (O) with (a) erythromycin $4 \mathrm{mg} / \mathrm{L}$, (b) tetracycline $4 \mathrm{mg} / \mathrm{L}$, and (c) ciprofloxacin $4 \mathrm{mg} / \mathrm{L}$. 


\section{References}

1. Limb DI. Mycoplasmas of the human genital tract. Med Lab Sci $1989 ; 46$ : 146-156.

2. Mårdh P-A. Human respiratory tract infections with mycoplasmas and their in vitro susceptibility to tetracyclines and some other antibiotics. Chemotherapy 1975; 21 Suppl 1 $47-57$.

3. Christiansson A, Mårdh P-A. Tetracycline-resistance in Mycoplasma hominis. Sex Transm Dis 1983; 10 Suppl : 371-373.

4. Evans RT, Taylor-Robinson D. The incidence of tetracyclineresistant strains of Ureaplasma urealyticum. J Antimicrob Chemother 1978; 4: 57-63.

5. D'Eustachio AJ, Johnson DR. Adenosine triphosphate content of bacteria. Fed Proc 1968; 27 : 761 (abstr)

6. Wheat PF, Hastings JGM, Spencer RC. Rapid antibiotic susceptibility tests on Enterobacteriaceae by ATP bioluminescence. J Med Microbiol 1988; 25: 95-99.

7. Wheat PF, Spencer RC, Hastings JGM. A novel luminometer for rapid antimicrobial susceptibility tests on gram-positive cocci by ATP bioluminescence. J Med Microbiol 1989; 29 . 277-282.

8. Kenny GE, Cartwright FD, Roberts MC. Agar dilution method for determination of antibiotic susceptibility of Ureaplasma urealyticum. Pediatr Infect Dis 1986; 5 Suppl 6: S332-S334

9. Limb DI, Wheat PF, Hastings JGM, Spencer RC. Antimicrobial susceptibility testing of Mycoplasma hominis by ATP bioluminescence. In : Stanley PE, McCarthy BJ, Smither $\mathrm{R}$ (eds) Adenosine triphosphate luminescence. Rapid methods in microbiology. London, Blackwell Scientific. 1989: 221-227.

10. Kenny GE, Hooton TM, Roberts MC, Cartwright FD, Hoyt J. Susceptibilities of genital mycoplasmas to the newer quinolones as determined by the agar dilution method. Antimicrob Agents Chemother 1989; 33: 103-107.

11. Stemler ME, Stemke GW, Robertson JA. ATP measurements obtained by luminometry provide rapid estimation of Ureaplasma urealyticum growth. J Clin Microbiol 1987; 25: 427-429.

12. Diver JM, Wise R. Morphological and biochemical changes in Escherichia coli after exposure to ciprofloxacin. J Antimicrob Chemother 1986; 18 Suppl D: $31-41$.

13. Oxley KM, Wheat PF, Spencer RC, Hastings JGM. Kinetics of antibiotic action studied using ATP bioluminescence. In: Schölmerich J (ed) Bioluminescence and chemiluminescence. Proceedings of the 4th International Symposium. Chichester, John Wiley. 1987: 495-498. 